\title{
CONTRAIL FORMATION IN THE TROPOPAUSE REGION CAUSED BY EMISSIONS FROM AN ARIANE 5 ROCKET
}

\author{
Ch. Voigt ${ }^{1,2}$, U. Schumann ${ }^{1}$, and K. Graf ${ }^{1}$ \\ ${ }^{1}$ Deutsches Zentrum für Luft- und Raumfahrt (DLR) \\ Institut für Physik der Atmosphäre \\ Oberpfaffenhofen, Wessling D-82234, Germany \\ ${ }^{2}$ Johannes-Gutenberg-Universität Mainz \\ Institut für Physik der Atmosphäre \\ 21 Johannes-Joachim-Becher-Weg, Mainz D-55099, Germany
}

Rockets directly inject water vapor and aerosol into the atmosphere, which promotes the formation of ice clouds in ice supersaturated layers of the atmosphere. Enhanced mesospheric cloud occurrence has frequently been detected near 80-kilometer altitude a few days after rocket launches. Here, unique evidence for cirrus formation in the tropopause region caused by ice nucleation in the exhaust plume from an Ariane 5-ECA rocket is presented. Meteorological reanalysis data from the European Centre for Medium-Range Weather Forecasts show significant ice supersaturation at the 100-hectopascal level in the American tropical tropopause region on November 26, 2011. Near 17-kilometer altitudes, the temperatures are below the Schmidt-Appleman threshold temperature for rocket condensation trail formation on that day. Immediately after the launch from the Ariane 5-ECA at 18:39 UT (universal time) from Kourou, French Guiana, the formation of a rocket contrail is detected in the high resolution visible channel from the SEVIRI (Spinning Enhanced Visible and InfraRed Imager) on the METEOSAT9 satellite. The rocket contrail is transported to the south and its dispersion is followed in SEVIRI data for almost $2 \mathrm{~h}$. The ice crystals predominantly nucleated on aluminum oxide particles emitted by the Ariane 5-ECA solid booster and further grow by uptake of water vapor emitted from the cryogenic main stage and entrained from the ice supersaturated ambient atmosphere. After rocket launches, the formation of rocket contrails can be a frequent phenomenon under ice supersaturated conditions. However, at present launch rates, the global climate impact from rocket contrail cirrus in the tropopause region is small. 


\section{INTRODUCTION}

Combustion of solid $\mathrm{NH}_{4} \mathrm{ClO}_{4} / \mathrm{Al}$ material in rocket propulsion systems leads to the emission of solid particles into the atmosphere, predominantly consisting of $\mathrm{Al}_{2} \mathrm{O}_{3}$. In addition, water vapor is injected by solid rocket boosters and, to a larger extent, by the cryogenic main stage of the rocket. In the atmosphere, the emission products in the initially hot and humid rocket exhaust plume mix with ambient air. Thereby, the exhaust cools down and the relative humidity in the plume increases. Eventually, the plume reaches saturation conditions with respect to liquid water in cold regions of atmosphere and the solid or processed coated plume particles may get activated into small water droplets. Ice nucleates in the activated water droplets as long as the plume is ice supersaturated. The initially small ice particles grow by uptake of ambient water or by aggregation with other ice particles. In addition, the plume particle concentration dilutes due to entrainment of ambient air. If the ambient air is supersaturated with respect to ice, the ice particles survive and grow further. If the ambient air is subsaturated with respect to ice, the rocket contrail particles may sublimate. The remaining solid $\mathrm{Al}_{2} \mathrm{O}_{3}$ aerosol can be transported over long distances and may modify ice clouds during their life cycles until they are finally removed from the atmosphere by rain out or snow out processes.

In the following, observations of aerosol in rocket plumes are summarized and evidence on ice cloud formation in the mesosphere related to the launch of space vehicles is compiled. Then, for the first time, a unique observation of contrail formation at altitudes below $20 \mathrm{~km}$ in the exhaust plume from an Ariane 5-ECA rocket from the SEVIRI onboard the geostationary satellite Meteosat-9 is presented. The ice formation process and follow the cirrus evolution over $2 \mathrm{~h}$ in the satellite data are discussed. Finally, the effects of rocket emissions and cirrus formation on atmospheric composition and climate are discussed.

\section{AEROSOL OBSERVATIONS IN ROCKET EXHAUST PLUMES}

Exhaust plumes from several rocket engines including a space shuttle launch vehicle, a Titan IV, an Athena II, an Atlas II, and a Delta II rocket have previously been probed with instruments on the NASA WB57 high altitude research aircraft and are compiled by Voigt et al. [1]. The $\mathrm{Al}_{2} \mathrm{O}_{3}$ particles with number concentrations of several hundred cubic centimeters in the size range of 0.01 to $4 \mu \mathrm{m}$ have been observed in 5-minute old stratospheric exhaust plumes from two solid rocket motors (SRM) from space shuttle launch vehicles and a Titan IV rocket [2]. The observations show a trimodal particle size distribution with mode mean diameters near $0.005,0.1$, and $2 \mu \mathrm{m}$. Similar aerosol concentrations have been measured in the stratospheric exhaust plume from a Delta II 
rocket powered by a combination of solid $\mathrm{NH}_{4} \mathrm{ClO}_{4} / \mathrm{Al}$ and liquid $\mathrm{LOx} /$ kerosene propulsion system [3].

Particle number, size, and composition measurements have been performed in the stratospheric exhaust plume from a small Athena II SRM at less than 30-minute plume age [4]. The plume contained alumina particles with number densities near 10000 per $\mathrm{cm}^{3}$ in the size range between 0.004 and $1.2 \mu \mathrm{m}$. More than 2000 exhaust particles from the Athena II SRM plume and the plume from the space shuttle launch vehicle have been analyzed with an aerosol mass spectrometer [5]. The rocket aerosol contained primary and trace components of the aluminum fuel and the combustion catalyst. In addition, chlorine from the oxidizer, iron, and elemental carbon were detected in the Athena II rocket plume and nitrate, phosphate, and water in the space shuttle plume particles. These observations suggest that the particle composition in the exhaust plumes is very diverse and that solid plume particles may get activated or coated with condensable exhaust products [5]. The detection of components of the rocket fuel, oxidizer, binding agents, and the combustion catalyst in the aerosol particles or on particle surfaces poses questions related to the reactivity and heterogeneous chlorine chemistry on coated alumina particles.

Enhanced concentrations of reactive nitrogen species (NOy) have also been observed in the Athena II SRM plume [6]. High nitric acid mixing ratios indicate that $\mathrm{HNO}_{3}$ is a significant plume component, most probably formed by the heterogeneous reaction of $\mathrm{ClONO}_{2}$ and $\mathrm{HCl}$ on the emitted alumina particles. High nitric acid concentrations at temperatures below $215 \mathrm{~K}$ may favour the formation of solid nitric acid trihydrate (NAT) particles in rocket exhaust plumes [7]. The NAT formation on meteoritic smoke or ice particles has frequently been observed in the polar stratosphere [8]. Release and formation of $\mathrm{HNO}_{3}$ in rocket exhaust plumes may cause supersaturation with respect to NAT and NAT nucleation on $\mathrm{Al}_{2} \mathrm{O}_{3}$ particles released by the SRM.

The injection of chlorine species as combustion product from the $\mathrm{NH}_{4} \mathrm{ClO}_{4} / \mathrm{Al}$ fuel and their activation by sunlight promotes ozone depletion in the plume. Complete ozone loss probably initiated by heterogeneous activation of chlorine species on solid or liquid plume particles or at warmer temperatures by gas phase reactions of the catalytic $\mathrm{ClO}$ dimer cycles has been observed in the Delta II rocket plume [3]. In addition, heterogeneous reactions on solid NAT or ice particles in the exhaust plume particles may enhance ozone loss.

The aerosol emissions from rocket exhaust plumes in the stratosphere can be compared to the meteoritic influx as natural source of particles into the stratosphere [1]. Most of the meteoritic mass influx in the atmosphere of 8 to $30 \mathrm{Gg}$ per year ablates at altitudes above $80 \mathrm{~km}$ due to frictional heating (see [9] and references therein). The ablation products condense, coagulate in the mesosphere and form nanometer-sized smoke particles. These meteoritic smoke particles are transported with the atmospheric circulation and sediment down. They remain about a year in the stratosphere, become well-mixed and partly incorporated 
into stratospheric sulfate aerosol [9]. The total rocket propellant emissions from a fleet of space shuttles, Titan IVs, Protons, Long Marchs, Ariane5s, Soyuz, Zenits, and smaller rocket launch vehicles averaged over 2000 to 2002 have been calculated to amount to about $30 \mathrm{Gg}$ per year [10]. About $9 \mathrm{Gg}$ per year are emitted into the stratosphere. The particulate alumina fraction of the rocket emissions in the stratosphere is about one third of the stratospheric emissions and amounts to $3 \mathrm{Gg}$ annually; hence, the solid particle rocket influx to the stratosphere is smaller than meteoritic influx [1].

\section{POLAR MESOSPHERIC CLOUD FORMATION AFTER ROCKET LAUNCHES}

Enhanced polar mesospheric cloud (PMC) formation has been detected after the launch of several small rockets and large space shuttle launch vehicles, as summarized in [1]. An increase in the mesospheric water concentration caused by rocket emissions has been measured with the Sounding of the Atmosphere with Broadband Emission Radiometry (SABER) instrument on the Thermosphere Ionosphere Mesosphere Energetics and Dynamics (TIMED) satellite [11]. In 2002, the radiometer detected radiance enhancements in altitudes of 90 and $130 \mathrm{~km}$ related in time and location to emissions from space shuttles or from liquid fuelled rockets. Almost half of the total liquid fueled launches, including all space shuttle launches, were identified in the radiance data.

An Arctic mesospheric $\mathrm{OH}$ layer has been observed a day after a space shuttle launch in 1997 above 85-kilometer altitude [12]. OH is produced predominantly by the photolysis of water vapor at these altitudes; hence, $\mathrm{OH}$ levels are an indicator for water vapor. About $300 \mathrm{Gg}$ of water vapor are injected into the altitude region between 108 and $114 \mathrm{~km}$ by rocket emissions [12], corresponding to water vapor mixing ratios of $3.5 \mathrm{ppmv}$ in these altitudes. One week after the space shuttle launch, a polar mesospheric cloud has been detected at 70 to $75^{\circ} \mathrm{N}$ latitudes with the CRyogenic Infrared Spectrometers and Telescopes for the Atmosphere-Shuttle PAllet Satellite (CRISTA-SPAS) instrument on the Discovery payload [12]. Trajectory calculations link the PMC observation site to the initial water vapor enhancement by the space shuttle exhaust. Hence, the observations suggest that rocket exhaust plumes can significantly perturb the mesospheric water vapor and cloud budget.

Transport of water vapor from a space shuttle exhaust plume to the southern hemisphere has been observed by the Global Ultraviolet Imager on the TIMED satellite [13]. Mesospheric water vapor emissions from the Columbia space shuttle launched from Kennedy Space Center in January 2003 were followed over 2 days, initially moving north-east. After one day, they changed to southern directions reaching $35{ }^{\circ} \mathrm{S}$. Ground-based light detection and ranging (lidar) observations from Rothera, Antarctica, show iron layers near 112-kilometer 
altitude 3 to 4 days after the space shuttle launch. As there is no known natural source of neutral iron above $100 \mathrm{~km}$, these observations have been interpreted as iron ablated from the shuttle's main engines.

Lidar, radar, and optical observations have also been performed in Alaska in the polar summer mesosphere shortly after a space shuttle launch in 2007 [14]. An exceptionally intense mesospheric cloud has been detected 3 days after the launch of the space shuttle. Similar to previous observations, the space shuttle launch was followed by enhanced polar mesospheric cloud formation and the buildup of an iron layer. The observed iron layer increased by a factor of 20 in density compared to background levels. Colocated with the iron layer, the radar records detected polar mesospheric summer echoes in this event.

A water vapor plume leading to the formation of an exceptionally bright PMC has been observed in a variety of satellite and ground based data sets as result of emissions from the main engines from the space shuttle's final launch in July 2011 [15]. Rocket emissions might explain the brightest fraction of the PMC population and interestingly larger ice particles were found above the smaller ones within the PMC.

Rocket exhaust trails are sensitive indicators for mesospheric dynamics such as vortex structures, wind shears, and turbulence. Emissions from two Soyuz rockets were used to examine the dynamical state of the mesosphere. Highly dynamic conditions were found to prevail in the mesosphere in May 2009, and the launch of the Soyuz created a shock wave, while exceptionally stable atmospheric conditions prevailed in the summer mesosphere in June 2011 [16].

Theoretical studies investigate the condensation and the sublimation [17] of ice crystals in the exhaust plumes from rocket emissions in the upper atmosphere. Solid particles may form in the upper atmosphere through condensation of water vapor and carbon dioxide released from engines of liquid fuelled rockets [18]. In thermal equilibrium, the ice crystals may grow to sizes $<2 \mathrm{~nm}$. In addition, taking into account the effects of particle heating caused by release of latent heat due to condensation, radiative heating, and energy losses to emission can explain ice layer thicknesses on particles $>7 \mathrm{~nm}$ [18]. In the following, ice formation processes in rocket exhaust plumes at lower altitudes in the tropopause region are theoretically investigated. First, let discuss the threshold temperature, under which ice particle can nucleate in rocket contrails.

\section{THEORETICAL ANALYSIS OF ROCKET EXHAUST WATER CONDENSATION TRAIL FORMATION IN THE TROPOPAUSE REGION}

Water condensation trails (contrails) form according to thermodynamics when the mixture between the warm and humid rocket exhaust gases and the cold but (in absolute humidity) relatively dry ambient air reaches liquid saturation. Let 
assume that this theory, developed for aircraft contrails, applies also to rocket exhaust, though the underlying concepts and saturation pressure values may well be limited to the range of tropospheric temperatures. The so-called SchmidtAppleman criterion requires that the ambient atmosphere must be cooler than a threshold temperature $T_{\mathrm{LC}}$ (for liquid condensation) which is computed as described by Schumann [19]. The threshold temperature is higher the larger the slope of the mixing line, $G=\partial e / \partial T$, of the water vapor partial pressure $e$ vs. temperature $T$ and pressure $p$ :

$$
G=\frac{c_{p} p \mathrm{EI}_{\mathrm{H}_{2} \mathrm{O}}}{\left(M_{\mathrm{H}_{2} \mathrm{O}} / M_{\text {air }}\right) Q_{\text {fuel }}(1-\eta)},
$$

with the specific heat capacity of air $c_{p}=1004 \mathrm{~J} /(\mathrm{kg} \cdot \mathrm{K})$, the ratio of molecular masses of water and air $M_{\mathrm{H}_{2} \mathrm{O}} / M_{\text {air }}=0.622$, the ambient pressure $p$ (in $\mathrm{Pa}$ ), the water vapor emission index $\mathrm{EI}_{\mathrm{H}_{2} \mathrm{O}}(\mathrm{kg} / \mathrm{kg})$, and the combustion heat $Q_{\text {fuel }}$ $(\mathrm{J} / \mathrm{kg})$ of the fuel used. The overall propulsion efficiency $\eta$ measures the fraction of combustion heat consumed to perform work by propulsion. The appearance of pressure $p$ implies decreases of threshold temperature with altitude. The parameter $\eta$ depends on the work performed by the rocket against friction, gravity, and mass acceleration relative to the amount of combustion heat released $(Q$ times fuel flow rate). The heat and kinetic energy of the exhaust gases represents energy losses not available for propulsion. The friction work contributes to warming the boundary layer air next to the rocket that mixes with the exhaust behind the rocket, earlier or later. Hence, this part joins the energy losses at least at some distance behind the rocket. However, the work performed by rocket mass acceleration and by altitude gain (potential energy) cannot heat the exhaust. Its computation requires detailed information on the rocket performance, which is not available to the authors.

For low orbits (e.g., $300 \mathrm{~km}$ ), the potential energy is only about $10 \%$ of the kinetic energy required to bring a rocket into orbit (i.e., to a speed of about $7900 \mathrm{~m} / \mathrm{s}$ ). The propulsion efficiency is smaller than for jet aircraft (0.3-0.4), because the rocket is losing kinetic energy with the mass burnt. Hence, let compute a lower bound of the threshold temperature for $\eta=0$ and a possible upper estimate for $\eta=0.2$.

According to information from the European Space Agency (ESA), the first stage Ariane 5 cryogenic rocket motor burns about $325 \mathrm{~kg} / \mathrm{s}$ of liquid hydrogen and liquid oxygen up to an altitude of about 205-kilometer altitude. This fuel consists of $84 \%$ mass fraction of $\mathrm{O}_{2}$ and $16 \%$ of $\mathrm{H}_{2}$. The exhaust contains $6.2 \%$ (mass) of unburnt $\mathrm{H}_{2}$ and $93.8 \%$ (mass) of water vapor. The solid boosters work up to an altitude of $140 \mathrm{~km}$ and burn up to about $4600 \mathrm{~kg} / \mathrm{s}$. The fuel consists of $68 \%$ mass fraction of $\mathrm{NH}_{4} \mathrm{ClO}_{4}, 18 \% \mathrm{Al}$, and $14 \% \mathrm{HTPB}$ (hydroxylterminated polybutadiene, used to bind the fuel and oxidizing agent into a solid mass). The booster exhaust contains only $5.13 \%$ of water vapor, besides solid $\mathrm{Al}_{2} \mathrm{O}_{3}$ particles, and other gases including condensables like hydrochloric acid. 
Table 1 Rocket emission parameters and threshold temperatures for water condensation trail formation for different rocket fuels, altitudes, and overall propulsion efficiencies $\eta$

\begin{tabular}{|c|c|c|c|c|c|}
\hline \multirow{3}{*}{ Fuel } & \multirow{3}{*}{$\mathrm{EI}_{\mathrm{H}_{2} \mathrm{O}}$} & \multirow{3}{*}{$Q, \mathrm{MJ} / \mathrm{kg}$} & \multicolumn{3}{|c|}{ Threshold temperature, ${ }^{\circ} \mathrm{C}$} \\
\hline & & & At $17 \mathrm{~km}$ & & $\mathrm{~km}$ \\
\hline & & & $\eta=0$ & $\eta=0$ & $\eta=0.2$ \\
\hline Cryogenic & 0.938 & 16.8 & -56.2 & -44.3 & -42.0 \\
\hline Solid & 0.0513 & 6.8 & -72.9 & -63.1 & -61.2 \\
\hline
\end{tabular}

The corresponding parameters $E I_{\mathrm{H}_{2} \mathrm{O}}$ and $Q$ are given in Table 1, together with the computed threshold temperature for $\eta=0$. The threshold values are computed using saturation pressure from Sonntag [20]. The emission indices for water vapor equals the $\mathrm{H}_{2} \mathrm{O}$ exhaust mass fractions. The combustion heat values can be computed following Hess's law for given fuel composition with values for the heat of formation from chemical tables. The latent heat of water evaporation has to be subtracted because it is released after contrail formation. The ambient temperature of about $-81{ }^{\circ} \mathrm{C}$ was cooler than the minimum threshold temperature of $-73^{\circ} \mathrm{C}$ at 17 -kilometer altitude $(\sim 88 \mathrm{hPa})$ (see Table 1$)$. Hence, a contrail should have been formed by water condensation from both motors at this altitude.

The threshold temperatures for rocket contrail formation calculated from the Schmidt-Appleman criterion [19] vs. altitude are shown in Fig. 1 for cryogenic

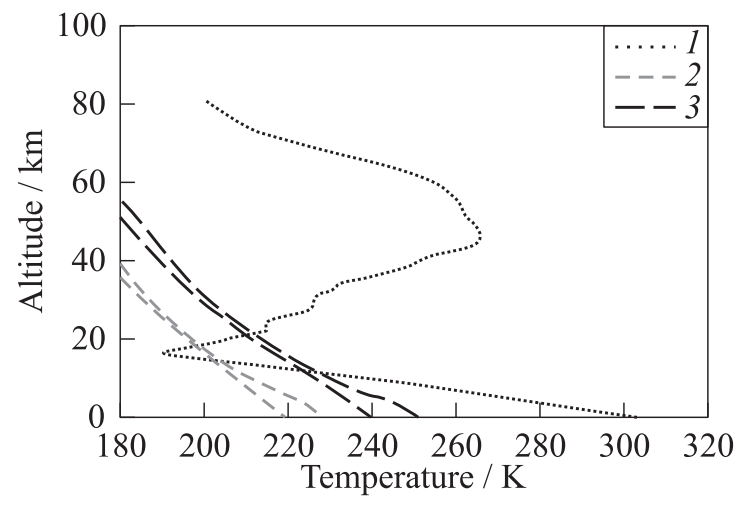

Figure 1 Threshold temperatures for rocket condensation trail formation for cryogenic liquid oxygen / hydrogen and SRM for different ambient relative humidity with respect to saturation over ice (solid curves - $\mathrm{RHi}=0 \%$ and dashed curves $\mathrm{RHi}=100 \%)$ and for zero propulsion efficiency $\eta=0$, together with the ambient temperature (1) from ECMWF reanalysis data above Kourou, French Guiana, on November 26,2010 at 18:00 UTC: 2 - solid; and 3 - cryogenic 
liquid oxygen / hydrogen and solid rocket motors and a lower limit of propulsion efficiency $\eta=0$. The temperature profile on November 26, 2010 is derived from meteorological reanalysis data from the European Centre for Medium-range Weather Forecast (ECMWF) up to 80-kilometer altitudes, corresponding to the upper model level at $1 \mathrm{hPa}$. The ECMWF integrates information from different observational sources such as ballons, sondes, aircraft and satellites, etc. into the data assimilation.

The ambient temperatures are below the threshold temperature for rocket contrail formation in the tropical tropopause region between 15 and $19 \mathrm{~km}$ for SRM emissions and between 11 and $20 \mathrm{~km}$ for cryogenic motor emissions. In the lower troposphere and the stratosphere, the temperatures are too warm for rocket contrail formation according the Schmidt-Appleman criterion.

Let now discuss the meteorological conditions on November 26, 2010 during the launch of an Ariane 5-ECA rocket in more detail. If the ambient atmosphere is supersaturated with respect to ice, rocket contrails can grow and become persistent. In contrast, under ice subsaturated conditions, ice crystals sublimate and contrails will not survive.

\section{METEOROLOGICAL CONDITIONS DURING THE LAUNCH FROM THE ARIANE 5-ECA ROCKET}

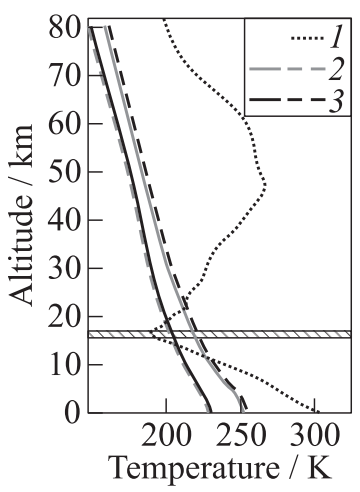

Figure 2 Vertical profile of temperature (1), threshold temperature for the formation of rocket contrails for different propulsion efficiencies (solid curves $-\eta$ $=0$ and dashed curves $-\eta=0.2): 2$ solid; and 3 - cryogenic
Vertical profiles of temperature, Schmidt-Appleman threshold temperature, water vapor, relative humidity, and horizontal wind velocities above Kourou, French Guiana, derived from ECMWF reanalysis data on November 26,2010 at $18 \mathrm{UTC}$ are given in Figs. 2 and 3 . Horizontal wind velocities near the tropopause are $10 \mathrm{~m} / \mathrm{s}$ in north-south and near $0 \mathrm{~m} / \mathrm{s}$ in eastwest directions. The atmosphere is supersaturated with respect to ice near the tropopause at 17-kilometer altitude and subsaturated with respect to ice above and below.

According to the SchmidtAppleman criterion, rocket ice crystals can nucleate in the tropical tropopause. Because of high ambient humidity with respect to ice, contrail 


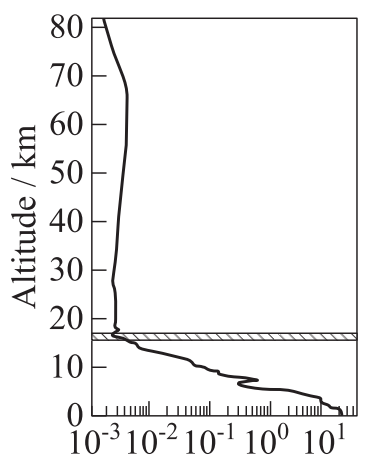

Specific humidity/ $\mathrm{g} / \mathrm{kg}$

(a)

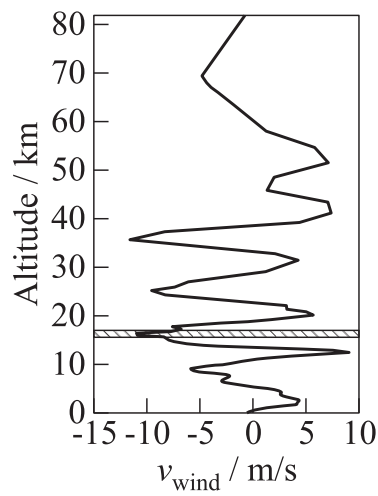

(c)

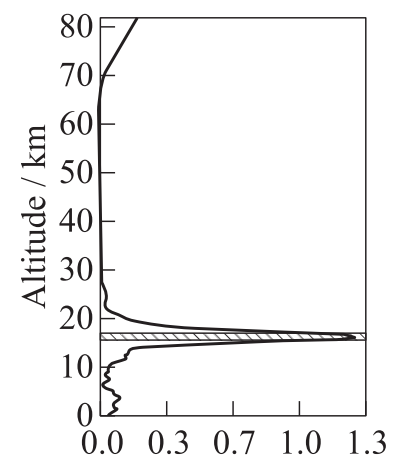

Relative humidity (ice)

(b)

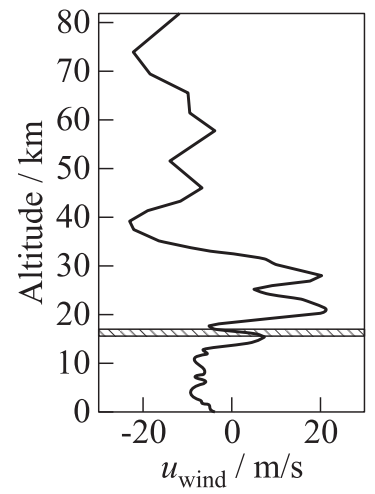

(d)

Figure 3 Specific humidity $(a)$, relative humidity with respect to ice $(b)$, and horizontal wind speeds $(c)$ on November 26, 2010 at 18:00 UTC from ECMWF reanalysis data

ice particles will grow and can get persistent. No contrails form at lower altitudes, because the atmosphere is too warm. In the stratosphere, the ambient temperatures are above the critical threshold temperature, again precluding contrail formation. In addition, contrail ice crystals would sublimate due to low ambient relative humidity.

Further, the horizontal relative humidity field at $100 \mathrm{hPa}$ from ECMWF data on November 26, 2010 will be shown (Fig. 4) to investigate rocket contrail growth. At 18:00 UTC (coordinate UT), the American tropical tropopause region is strongly supersaturated with respect to ice at the 100-hectopascal level. Hence, meteorological conditions favour the nucleation and growth of ice crystals near and below the tropopause on that day. 


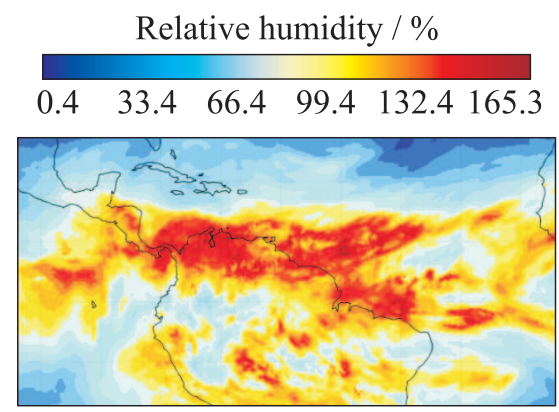

Figure 4 Relative humidity with respect to ice on November 26, 2010 at 18:00 UTC from ECMWF reanalysis data at the 100-hectopascal level

\section{SATELLITE OBSERVATIONS OF CONTRAIL FORMATION IN THE EXHAUST PLUME FROM THE ARIANE 5-ECA ROCKET}

While formation of ice particles related to rocket exhaust plumes has been reported previously in the mesosphere, let now discuss ice nucleation in rocket plumes in the tropopause region near 17-kilometer altitude. Ice can nucleate in the rocket exhaust, when the Schmidt-Appleman criterion is fulfilled [19]; hence, the ambient temperature is lower than a critical threshold temperature as given in Fig. 1. After the release of emissions into the atmosphere, the hot rocket exhaust mixes with ambient cold air and cools down. When saturation with respect to water is reached, water condenses predominantly on solid $\mathrm{Al}_{2} \mathrm{O}_{3}$ plume particles and forms small water droplets. Under ice saturated conditions, ice nucleates in the activated plume aerosol. During further contrail life time, the ice particles grow as long as the exhaust plume is supersaturated with respect to ice. The particle number density decreases due to entrainment of ambient air. The rocket contrail may survive for several hours and become persistent. During its life time, the rocket contrail cirrus loses its structure, gets entrained in ambient natural cirrus, and, hence, may modify natural cirrus cloudiness.

Here, the unique case of contrail formation in the tropopause region in the exhaust plume from an Ariane 5-ECA rocket is presented. The Ariane 5-ECA with the flight number V-198 (serial number 556) was launched on November 26, 2010 at 18:39 UTC at the Space Centre Kourou $\left(5^{\circ} 9^{\prime} \mathrm{N}, 52^{\circ} 39^{\prime} \mathrm{W}\right)$ in French Guiana to a geostationary transfer orbit. Its payload consisted of two telecommunication satellites (Intelsat 17 and HYAS 1) with a weight of $8867 \mathrm{~kg}$.

Here, an analysis of this launch using satellite data from the SEVIRI onboard Meteosat-9 satellite positioned in geostationary orbit at $0^{\circ}$ is performed. The SEVIRI instrument provides information on solar and terrestrial radiation in 12 
spectral channels. Eleven of the spectral channels have a resolution of $3 \mathrm{~km}$ at the subsatellite point while the High Resolution Visible (HRV) channel provides data with a resolution of $1 \mathrm{~km}$. In addition, the SEVIRI instrument has a high temporal resolution of $15 \mathrm{~min}$, which is necessary for the launch analysis and to investigate the cloud life cycle. As clouds and rocket plumes are the small-scale and fast moving objects, let employ the high temporal and spatial resolution pro-
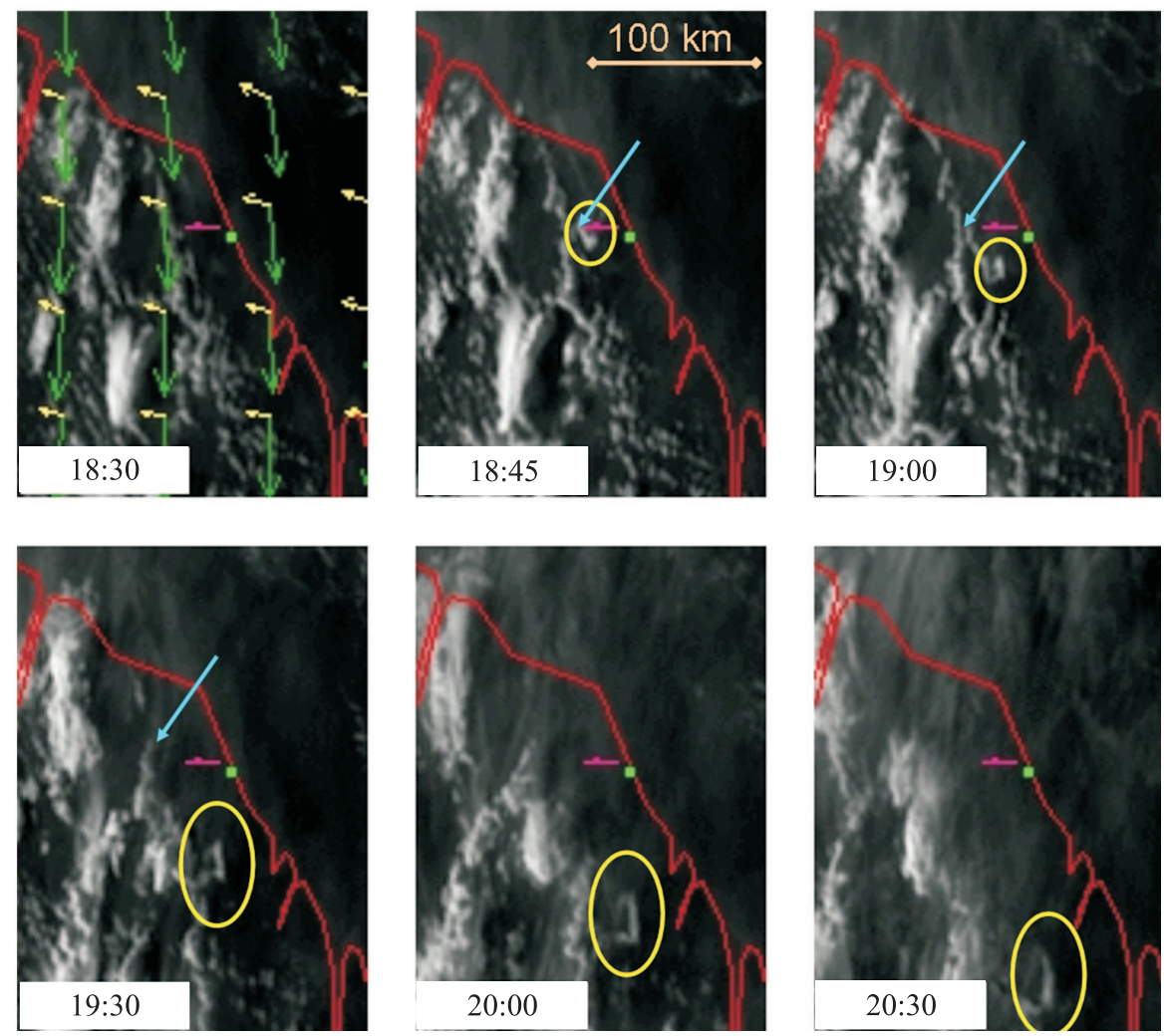

Figure 5 Satellite images from METEOSAT-9 SEVIRI instrument, HRV channel on November 26, 2010. All scenes: green dots indicate position of Kourou City in French Guyana. The pink line represents trajectory of the Ariane 5-ECA launch vehicle from 0- to 20-kilometer altitude. Whereas Ariane 5-ECA originally started eastwards, trajectory is seen westwards due to parallax effects. Scene at 18:30 UTC (before start): mainly natural cloudiness, wind direction in $850 \mathrm{hPa}$ (yellow arrows), wind direction in $100 \mathrm{hPa}$ (green arrows). Scenes at 18:45 UTC and thereafter: the yellow circles indicate position of the Ariane 5-ECA rocket contrail near 17-kilometer altitude. The blue arrows mark the position of the low-level rocket plume below 5-kilometer altitude 
vided by the HRV channel. The HRV channel is located in the visual spectrum; therefore, no information is available during night time.

Figure 5 shows 6 high resolution visible images at 15- or 30-minute time steps between 18:30 and 20:30 UTC on November 26, 2010. The location of the launch site Kourou is marked by a green dot. The Ariane 5-ECA flight track from the surface to 20-kilometer altitude is marked by a pink line in each image.

At 18:30 UTC, 9 min before the rocket launch, mainly natural cloudiness is detected by the satellite instrument. In $850 \mathrm{hPa}$ near the ground, the ECMWF wind direction is to the west (yellow arrows) and in $100 \mathrm{hPa}$, the wind direction is to the south (green arrows) (also, see Figs. 2 and 3). After the Ariane 5-ECA launch at 18:39 UTC, two effects of rocket emissions can be observed in the satellite images. At 18:45 UTC, a line-shaped cloud can be identified and related to the launch site and the rocket emissions. The line-shaped structure marked by the yellow circles drifts in southern direction. The rocket contrail is visible in each image within the next $2 \mathrm{~h}$.

In addition, there is an exhaust plume marked with the blue arrow drifting westwards with the wind direction at lower altitudes. The ECMWF wind fields at low altitudes are directed towards the west, hence, support the impression that the westward moving cloud structure (blue arrows) is caused by the particulate rocket exhaust plume near takeoff in altitudes below 2 to $5 \mathrm{~km}$. In contrast, the line-shaped contrail structure drifts in southern direction. The ECMWF reanalysis of wind fields show winds from north only in a small altitude range at $17 \mathrm{~km}$ (see Figs. 2 and 3), far above the level of local air traffic. Hence, the present study suggests that a rocket contrail had formed in the tropical tropopause region after the launch of the Ariane 5-ECA rocket on November 26, 2010. It grew, spread out, and was transported to the south for $2 \mathrm{~h}$.

\section{SUMMARY AND OUTLOOK}

Strong local and regional perturbations of the atmospheric aerosol have been detected by in situ instruments on aircraft after the launch of rockets mainly caused by the combustion of solid propellants. In addition, enhanced water vapor and iron levels followed by enhanced mesospheric cloud formation in polar and extrapolar regions of the mesosphere have frequently been observed after rocket and space shuttle launches. Here, atmospheric conditions for rocket contrail formation were investigated using the Schmidt-Appleman criterion and temperatures below which rocket contrail formation is possible were derived. The results were compared to the atmospheric conditions prevailing on November 26, 2010 in the tropical tropopause above the launch site Kourou in French Guiana. According to the Schmidt-Appleman criterion, rocket contrail formation is possible only in the upper troposphere / lower stratosphere region on that day.

Further, first direct evidence for ice formation in the tropopause region caused by rocket emissions will be presented. Data analysis from the SEVIRI instrument 
onboard the Meteosat-9 satellite show a linear-shaped ice cloud few minutes after the launch of an Ariane 5-ECA rocket on November 26, 2010. The tropopause region is ice-supersaturated; hence, the ice crystals grow and rocket contrail cirrus spreads out and is transported to the south. The present authors suggest that ice nucleation on solid or processed $\mathrm{Al}_{2} \mathrm{O}_{3}$ particle emissions is the dominant pathway for rocket contrail formation at these altitudes.

Rocket emissions are washed out within weeks in the troposphere, while the lifetime of rocket emissions in the stratosphere is a few years depending on emission altitude. Assuming similar optical and radiative properties of aircraft and rocket contrails, the local instantaneous radiative forcing of rocket contrails in the order of some $\mathrm{W} / \mathrm{m}^{2}$ was estimated. However, the integration over the globe as well as launch rates of 20 to 30 rockets per year yields significantly smaller global averages and the climate impact from rocket contrails in the tropopause region is small. Still, the rocket impact on climate could be different in higher altitudes in the mesosphere, where mesospheric cloud formation may modify the atmospheric radiation budget and amplify dynamical processes.

New in situ data on aerosol, humidity, and ice particle size distributions in rocket contrails combined with process modeling would be required to investigate ice nucleation in rocket contrails in more detail. Further, the observation of a rocket contrail in 17-kilometer altitude and the detection of a particulate rocket aerosol layer at ground levels from Meteosat SEVIRI images should be verified for other rocket launches from different launch sites in order to better quantify the local and global effects of rocket emissions on the atmosphere.

\section{REFERENCES}

1. Voigt, C., U. Schumann, K. Graf, and K.-D. Gottschaldt. 2013. Impact of rocket exhaust plumes on atmospheric composition and climate - an overview. Progress in propulsion physics. Eds. L. T. DeLuca, C. Bonnal, O. Haidn, and S. Frolov. EUCASS advances in aerospace sciences book ser. TORUS PRESS - EDP Sciences. 4:657-670. doi: 10.1051/eucass/201304657.

2. Ross, M. N., P. D. Whitefield, D. E. Hagen, and A. R. Hopkins. 1999. In situ measurement of the aerosol size distribution in stratospheric solid rocket motor exhaust plumes. Geophys. Res. Lett. 26:7-13. doi: 10.1029/1999GL900085.

3. Ross, M. N., P. D. Whitefield, D. E. Hagen, and A. R. Hopkins. 1999. Observation of stratospheric ozone depletion associated with Delta II rocket emissions. Geophys. Res. Lett. 27:15-19. doi:10.1029/1999GL011159.

4. Schmid, O., J. M. Reeves, J. C. Wilson, et al. 2003. Size-resolved particle emission indices in the stratospheric plume of an Athena II rocket. J. Geophys. Res. 108:4250-4256. doi: 10.1029/2002JD002486.

5. Cziczo, D. J., D. M. Murphy, D. S. Thomson, and M. N. Ross. 2002. Composition of individual particles in the wakes of an Athena II rocket and the space shuttle. Geophys. Res. Lett. 29:2047-2051. doi: 10.1029/2002GL015991. 
6. Popp, P. J., B. A. Ridley, J. A. Neuman, et al. 2002. The emission and chemistry of reactive nitrogen species in the plume of an Athena II solid-fuel rocket motor. Geophys. Res. Lett. 29:1887-18891. doi: 10.1029/2002GL015197.

7. Gates, A. M., L. M. Avallone, D.W. Toohey, et al. 2002. In situ measurements of carbon dioxide, $0.37-4.0 \mu \mathrm{m}$ particles, and water vapor in the stratospheric plumes of small rockets. J. Geophys. Res. 107:4649-4656. doi:10.1029/2002JD002121.

8. Voigt, C., J. Schreiner, A. Kohlmann, et al. 2002. Nitric acid trihydrate (NAT) in polar stratospheric clouds. Science 290:1756-1758.

9. Cziczo, D. J., D. S. Thomson, and D. M. Murphy. 2001. Ablation, flux and atmospheric implications inferred from stratospheric aerosol. Science 291:1772-1775.

10. Jackman, C., D. Considine, and E. Fleming. 1996. Space shuttles impact on the stratosphere: An update. J. Geophys. Res. 101(D7): doi:10.1029/96JD00577.

11. Siskind, D. E., M. H. Stevens, J. T. Emmert, et al. 2003. Signatures of shuttle and rocket exhaust plumes in TIMED/SABER radiance data. Geophys. Res. Lett. 30:1819-1823. doi:10.1029/2003GL017627.

12. Stevens, M. H., J. Gumbel, C. R. Englert, et al. 2003. Polar mesospheric clouds formed from space shuttle exhaust. Geophys. Res. Lett. 30:1546-1550. doi:10.1029/2003GL017249.

13. Stevens, M.H., R.R. Meier, X. Chu, M. T. DeLand, and J. M. C. Plane. 2005. Antarctic mesospheric clouds formed from space shuttle exhaust. Geophys. Res. Lett. 32:L13810. doi:10.1029/2005GL023054.

14. Kelley, M. C., M. J. Nicolls, R.H. Varney, et al. 2010. Radar, lidar, and optical observations in the polar summer mesosphere shortly after a space shuttle launch. J. Geophys. Res. 115:A05304. doi:10.1029/2009JA014938.

15. Stevens, M. H., S. Lossow, J. Fiedler, et al. 2012. Bright polar mesospheric clouds formed by main engine exhaust from the space shuttle's final launch. J. Geophys. Res. 117:D19206. doi:10.1029/2012JD017638.

16. Dalin, P., V. Perminov, N. Pertsev, et al. 2013. Optical studies of rocket exhaust trails and artificial noctilucent clouds produced by Soyuz rocket launches. J. Geophys. Res. 118. 14:7850-7863. doi:10.1002/jgrd.50549.

17. Platov, Y.V., and M. J. Kosch. 2003. Sublimation of ice particles from rocket exhausts in the upper atmosphere. J. Geophys. Res. 108. A12:1434. doi: 101029/2003JA009936.

18. Platov, Y. V., A.I. Semenov, and B. P. Filippov. 2011. Condensation of combustion products in the exhaust plumes of rocket engines in the upper atmosphere. Geomagnetism Aeronomy 51(4):550-556.

19. Schumann, U. 1996. On conditions for contrail formation from aircraft exhausts. Meteorol. Z. 5:4-23.

20. Sonntag, D. 1994. Advancements in the field of hygrometry. Meteorol. Z. 3:51-66. 